\title{
Lung and Gut Microbiota as Potential Hidden Driver of Immunotherapy Efficacy in Lung Cancer
}

\author{
Carmine Carbone, ${ }^{1,2}$ Geny Piro, ${ }^{1,2}$ Vincenzo Di Noia, ${ }^{1,2}$ Ettore D'Argento, ${ }^{1,2}$ \\ Emanuele Vita, ${ }^{1,2}$ Miriam Grazia Ferrara, ${ }^{1,2}$ Sara Pilotto $\left(\mathbb{D},{ }^{3}\right.$ Michele Milella, ${ }^{3}$ \\ Giovanni Cammarota $\left(\mathbb{D},{ }^{2,4}\right.$ Antonio Gasbarrini $\mathbb{D}^{2,4}$ Giampaolo Tortora, ${ }^{1,2}$ \\ and Emilio Bria ${ }^{1,2}$ \\ ${ }^{1}$ Comprehensive Cancer Center, Fondazione Policlinico Universitario Agostino Gemelli, IRCCS, Rome, Italy \\ ${ }^{2}$ Università Cattolica del Sacro Cuore, Rome, Italy \\ ${ }^{3}$ Section of Medical Oncology, Department of Medicine, University of Verona and University Hospital Trust, Verona, Italy \\ ${ }^{4}$ Digestive Disease Center, Policlinico Universitario Agostino Gemelli, IRCCS, Rome, Italy \\ Correspondence should be addressed to Emilio Bria; emilio.bria@unicatt.it
}

Received 22 May 2019; Revised 12 September 2019; Accepted 26 September 2019; Published 11 November 2019

Guest Editor: Ciriaco A. Piccirillo

Copyright (c) 2019 Carmine Carbone et al. This is an open access article distributed under the Creative Commons Attribution License, which permits unrestricted use, distribution, and reproduction in any medium, provided the original work is properly cited.

\begin{abstract}
Lung cancer is one of the deadliest and most common malignancies in the world, representing one of the greatest challenges in cancer treatment. Immunotherapy is rapidly changing standard treatment schedule and outcomes for patients with advanced malignancies. However, several ongoing studies are still attempting to elucidate the biomarkers that could predict treatment response as well as the new strategies to improve antitumor immune system response ameliorating immunotherapy efficacy. The complex of bacteria, fungi, and other microorganisms, termed microbiota, that live on the epithelial barriers of the host, are involved in the initiation, progression, and dissemination of cancer. The functional role of microbiota has attracted an accumulating attention recently. Indeed, it has been demonstrated that commensal microorganisms are required for the maturation, education, and function of the immune system regulating the efficacy of immunotherapy in the anticancer response. In this review, we discuss some of the major findings depicting bacteria as crucial gatekeeper for the immune response against tumor and their role as driver of immunotherapy efficacy in lung cancer with a special focus on the distinctive role of gut and lung microbiota in the efficacy of immunotherapy treatment.
\end{abstract}

\section{Introduction}

The small (SCLC) and non-small-cell lung cancer (NSCLC) (referred as lung cancer "LC" hereafter) is one of the deadliest malignancies in the world. For 2019, the American Cancer Society estimates 116,440 and 111,710 new LC cases with $24 \%$ and $23 \%$ of new deaths per year for men and women, respectively [1]. Over the past few decades, the research on genetics of LC improved the opportunity to select patients that could benefit from the most recent immune-based therapeutic strategies [2-8].

Several clinical trials established the efficacy of immunotherapy on different tumors bringing to the approval of this new therapeutic regimen. The clinical trials CheckMate 017, CheckMate 057, and Keynote 010 demonstrated that the monoclonal antibodies (mAbs) against programmed cell death-1 (PD-1) nivolumab [9] and pembrolizumab [10] significantly improved the overall survival (OS) over docetaxel in NSCLC patients after the failure of prior platinum-based chemotherapy. Similarly, the OAK trial showed that atezolizumab [11], an anti-PD-ligand 1 (PD-L1) mAb, produced a survival benefit compared with docetaxel in the same NSCLC population. In details, the anti PD-(L)1 therapy blocks the binding of PD-1 to its ligand (PDL-1) restoring the functions of "exhausted" T cells and resulting in tumor shrinkage [12]. The immunoblocking between PD-1 and activated cytotoxic 
T lymphocytes (CTLs), and between PD-L1 and tumor cells, has exhibited significant clinical efficacy in different types of cancer and was currently approved for treating tumors, including advanced stage of NSCLC [13]. Consistently, nivolumab and pembrolizumab showed impressive efficacy also in SCLC [14].

Actually, five monoclonal antibodies targeting immune checkpoints have been approved by the U.S. Food and Drug Administration (FDA) for cancer treatment alone or in combination with platinum-based chemotherapy [9], although ongoing study attempts to discover new predictive biomarker of treatment response as well as new strategies to improve immunotherapy efficacy, including the combination of antiPD-(L) 1 and anti-Cytotoxic T Lymphocyte Antigen 4 (CTLA4) agents $[15,16]$.

Several studies demonstrated that the gut microbiome regulates the power by which immunotherapy may stimulate the anticancer immune response (reviewed in [17]).

Commensal microorganisms are required for the maturation, education, and function of the immune system. A tight and continuous interaction of immune cells with microorganisms allows learning the difference between commensal and pathogenic bacteria. Indeed, the haematopoietic and nonhaematopoietic cells of the innate immune system are strategically located at the host-microbiome interface and are rich of pattern recognition receptors (PRRs) that sense microorganism presence [18]. This relationship leads to the concept of humans as mammalian holobionts resulting from parallel coevolution of host-eukaryotic and microbe-prokaryotic elements.

The gastrointestinal tract hosts are the most abundant and diversified microbial population. The gut microbiota is composed of $10^{13}$ to $10^{14}$ microorganisms whose genome is collectively at least 100 times the human genome [19]. Moreover, behind gut epithelia, bacteria colonize other specialized epidermal surfaces like the ductal system of exocrine organs and respiratory tract.

The human respiratory tract is the main portal of entry for numerous microorganisms. Interestingly, gut and lung microbiota are connected by a complex bidirectional axis via lymphatic [20] and blood circulation, and modification of one mucosal compartment can directly impact distant mucosal site [21].

Recent high-depth metagenomic sequencing techniques have changed our understanding of the complex microbiome ecosystem enabling the identification and quantification of individual bacterial strains and the correlation between specific microbiome asset and disease status. More interesting, wide efforts are now focused on how variations in these populations may influence response to immunotherapy.

In this review, we discuss some of the major findings depicting bacteria as crucial gatekeeper for the immune response against tumor and their role as driver of immunotherapy efficacy in lung cancer.

\section{Role of Commensal Bacteria in Cancer Response to Immunotherapy}

During early life, the immune system is broadly stimulated with the first contact to microorganisms at gastrointestinal and lung barriers [22]. This primary wave of microbial exposure exerts a long-lasting effect on immune cell function [23].

Increasing evidence supports the idea of a dynamic interaction between immune cells, microbiota, and tumor microenvironment. Gene expression analysis of tumors from antibiotic-treated mice showed a downregulation of genes related to inflammation, phagocytosis, antigen presentation, and adaptive immune response. Moreover, microbiota disruption impairs the efficacy of CpG-oligonucleotide immunotherapy affecting myeloid-derived cell functions in the tumor microenvironment [24].

Furthermore, it has been demonstrated that oral administration of Bifidobacterium improves response to anti-PDL1 antibody in mouse models of cancer by inducing dendritic cell function and increasing $\mathrm{CD}^{+} \mathrm{T}$ cell accumulation in the tumor microenvironment [25]. Microbiota composition has also a key role in the immunostimulatory effects of Cytotoxic T-Lymphocyte Antigen 4 (CTLA-4) blockade. In details, Bacteroides species affect interleukin- (IL-) 12-dependent Th1 immune response facilitating tumor control in mice and patients [26].

A recent study analyzed baseline stool samples from 42 metastatic melanoma patients before immunotherapy treatment demonstrating an abundance of Bifidobacterium longum, Collinsella aerofaciens, and Enterococcus faecium in responding patients. Fecal transplantation of germ-free mice with stool from responding patients improved efficacy of anti-PD-L1 therapy increasing immune-mediated tumor control through the induction of $\mathrm{T}$ cell response [27].

The different microbiota composition between cancer patients and healthy individuals not only demonstrated diagnostic and prognostic potentials of special microbial pathogens in cancer but also suggested the idea that the manipulation of the microbiota could be a valid approach for a better therapeutic response, acting on drug efficacy or enhancing the immune system (discussed below).

The fecal microbiota transplantation (FMT) (i.e., the transfer of fecal bacteria from a donor into a recipient) that has been applied to clinical practice for the treatment of Clostridium difficile infection [28], ulcerative colitis [29-31], and irritable bowel syndrome [32] demonstrated an effect also on the systemic immune response and particularly on the mechanisms of immune surveillance against LC (Routy et al.). Routy and colleagues demonstrated that a specific host gut microbiota might contribute to patient immunotherapy response. Antibiotic-induced alterations of gut microbiota during immunotherapy treatment dampens patient response to the therapy. Interestingly, the FMT from patients sensitive to immunotherapy is able to revert the immunotherapy response in treatment-resistant patients. These findings lead to intriguing hypothesis that the modification of gut microbiota through FMT could enhance the response also in tumors resistant to immunotherapy.

The overall results of these studies open the avenue to propose a multiparameter prediction model integrating conventional parameters, such as tumor genetic alterations, with microbiota assessment to select patients most likely to respond to immunotherapies. 


\section{Effects of Gut Microbiota on LC}

The role of the human gut microbiome is being increasingly accepted. From 2015 to present, more than 158 papers on high-impact journals were published and several research groups indicated the role of the gut microbiome in different diseases with a particular emphasis on cancers (https://www.ncbi.nlm.nih.gov/pubmed?term $=($ LUNG\%20 CANCER\%20MICROBIOME)\%20AND\%20(\%222015\%2F0 $1 \% 2$ F01\%22\%5BDate $\% 20$-\%20Publication $\% 5$ D $20 \% 3 \mathrm{~A} \%$ 20\%223000\%22\%5BDate\%20-\%20Publication\%5D).

More than 100 trillion bacteria colonize the human intestines [33]. The crosstalk between the gut microbiota and the immune system contributes to the health status of the host. The application of this concept in oncology field is particularly important, and several recent papers highlighted the role of gut microbiota as one of the regulatory factors affecting both the tumor proliferation and the immunological environment of cancer, determining thus the efficacy of the treatment with the immune checkpoint inhibitors. The specific role of gut microbiota in supporting cancer development and growth is yet unclear. However, there are compelling evidences of the gut microbiota role in modulating both innate and adaptive immune response and how this influences tumor growth and immune escape [34]. Moreover, the gut microbiota is able to regulate host immunity both locally and at distal sites [35] modulating the expansion and differentiation of $\mathrm{T}$ cell populations. Briefly, the pathogenassociated molecular patterns (PAMPs) of the microorganisms in the intestines are recognized by the Toll-likereceptors (TLRs) on the membrane of intestinal epithelial cells. The activation of TLRs leads to the activation of signal cascade that finally results into the stimulation of immunological cells in the lamina propria. Dendritic cells and macrophages, activated in mesenteric lymph nodes (MLN), prime the naïve $\mathrm{B}$ and $\mathrm{T}$ cells to mature and differentiate, producing, thus, IgA. Differentiated $\mathrm{T}$ cells assume both profile of Th1 and/or Th17 proinflammatory cells activating additional effector cells as neutrophils or anti-inflammatory cells to control immune response [36-43]. Moreover, high diversity of gut microbiome supports M1 macrophage and Th1 lymphocyte differentiation, activation of helper/cytotoxic T cell, and upregulation of PD-1 expression on lymphocytes [44].

All these studies highlighted the potential of gut microbiota manipulation in cancer treatment, especially in tumors where the immunotherapy is currently adopted in clinical practice such as the LC and melanoma.

In melanoma, PD-1 inhibitors produce long-lasting responses in 30-40 percent of patients. However, these drugs do not work in the other 60-70 percent of melanoma patients for a multitude of reasons, including not having the right microbes in the gut-a condition termed "intestinal dysbiosis." Likewise, several phase III LC clinical trials revealed that immunoblockade treatment leads to only approximately $20 \%$ of patients' overall objective response (OOR) and that median duration of response is significantly heterogeneous [45-47]. Recent studies demonstrated that gut microbiota could modulate immunotherapy response. Indeed, gut com- mensals such as $B$. thetaiotaomicron or $B$. fragilis are predictive factors for anti-CTLA- 4 treatment in a mouse melanoma model [26].

It is therefore desirable to identify patients who would benefit more from immunotherapy and to understand what drives resistance in the patients who do not respond.

A study by Routy et al. proved that the gut microbiota plays a critical role in the response to $\mathrm{PD}-1$ blockade and may have a prognostic value in LC. Moreover, the gut microbiota of patients who respond to immunotherapy with checkpoint inhibitors was different from those who do not. In particular, the authors identified an increased level of Akkermansia muciniphila (A. muciniphila) in patients who experienced longer survival. They demonstrated that gut microbiota not only was a predictor of response but also regulated the efficacy of anti-PD1 in murine models. In fact, the fecal microbiota transplantation from responder mice restored PD-1 blockade sensibility in the same models. Interestingly, the authors demonstrated that gut microbiome, and in particular A. muciniphila, influences efficacy of PD-1-based immunotherapy against epithelial tumors increasing the presence of tumor-infiltrated $\mathrm{CCR} 9^{+} \mathrm{CXCR} 3^{+} \mathrm{CD} 4^{+} \mathrm{T}$ cells through a IL-12-dependent signaling pathway [48].

A recent paper using data from 37 advanced NSCLC patients receiving nivolumab enrolled in the study from the clinical trials CheckMate 078 (NCT02613507) and CheckMate 870 (NCT03195491) demonstrated a strong correlation between the level of gut microbiome diversity and anti-PD-1 efficacy in advanced NSCLC Chinese patients. The patients with high gut microbiome diversity (reported as favorable gut microbiome) exhibited an increase of memory $\mathrm{T}$ and NK cell signatures in the peripheral blood samples. These findings provide important implications for the prediction of anti-PD-1 immunotherapy response in Chinese population with NSCLC [49].

To date, a single study examined the association among antibiotics and efficacy of immune checkpoint inhibitors. In this retrospective analysis of the data from 90 NSCLC patients treated (13 patients) or untreated ( 77 patients) with antibiotics prior to nivolumab therapy as second or later line of therapy, the authors demonstrated that antibiotic treatment reduced significantly both Progression-Free Survival (PFS) and OS. Although, in multivariate analysis, no statistically significant association was found between survival and prior antibiotic use, a trend concerning the negative influence of antibiotic use was conveyed. These data, although need further validations, confirmed that gut microbiota could have an important role in shaping systemic immune responses [50].

Botticelli and colleagues demonstrated that a specific gut microbiome may influence the response to immunotherapy. In particular, by using the NGS technique, the authors showed that there are higher levels of Rikenellaceae, Prevotella, Streptococcus, Lactobacillus, Bacteroides plebeius, Oscillospira, and Enterobacteriaceae in the stool of NSCLC patients than in healthy controls. Moreover, patients who respond to nivolumab treatment had less abundance of Ruminococcus bromii, Dialister, and Sutterella spp. than not responders [51]. 
The concept of immunomodulatory ability is also applicable to the chemotherapy regimen able to regulate the immune system. Cyclophosphamide is well known for its antineoplastic and immunomodulating ability and was registered for early and advanced breast cancer. In a transgenic tumor mouse model of autochthonous lung carcinogenesis, this alkylating agent alters the composition of microbiota in the small intestine inducing translocation of specific Grampositive bacteria, including Lactobacillus johnsonii (growing in $>40 \%$ cases), Lactobacillus murinus, and Enterococcus hirae, into secondary lymphoid organs [52]. Here, the Gram-positive bacteria stimulate the generation of a specific subset of "pathogenic" T helper 17 (pTh17) cells and memory Th1 immune response. In germ-free or antibiotictreated animal models, the absence of these bacteria leads to a reduction in pTh17 response and cyclophosphamide tumor resistance. Adoptive transfer of pTh17 cells partially restored the antitumor efficacy of cyclophosphamide. These results suggest that the gut microbiota helps shape the anticancer immune response in LC patients [53].

\section{Effects of Lung Microbiota on LC}

The lung is constantly exposed to microorganisms from the air and the upper respiratory tract; therefore, it is not a "sterile place" as previously believed. Acquisition of lung microbiome is a crucial event in newborn to protect the lung from injuries [54]. Lung tissue hosts a unique microbiome asset with less diversity, compared to the intestinal one, but equally affected by drugs, disease, and eating habits, which can create a selective pressure on reproducing communities. The specific composition of the lung microbiome results from the balance of three phenomena: microbial immigration, microbial elimination, and the relative reproduction rates of its members [55].

Dysbiosis of lung microbiome ecosystem and the epithelial integrity loss in heavy smokers could be the initial cause of inflammation in chronic obstructive pulmonary disease and LC [56]. A comparative analysis of 142 LC patients and 33 healthy controls reveals a distinct lung microbiome profile associated with tumor tissue [57]. Moreover, epidemiological evidence indicates a significant association between prolonged antibiotic exposure and incidence of LC [58].

Exacerbations of chronic lung disease have shown correlation with microbiota disorder of the respiratory tract. Respiratory dysbiosis is closely linked to a dysregulated host immune system, which in turn further affects lung microenvironment promoting inflammation [59].

On the other hand, a recent study claims that depletion of local commensal microbiota or blockade of the downstream cellular/molecular immune mediators suppresses the development of lung adenocarcinoma. By using the conditionally genetically engineered mouse model (GEMM) of lung adenocarcinoma, the authors demonstrated that commensal bacteria stimulate production of IL- $1 \beta$ and IL- 23 from myeloid cells via a Myd88-dependent pathway. This event leads to proliferation and activation of tissue resident $\gamma \delta \mathrm{T}$ cells with a consequent increased production of effector molecules, such as IL-17, to promote inflammation and tumor cell proliferation [60]. However, this study does not deep investigate the specific strain composition of the lung microbiota responsible for lung tumor development.

Many efforts have been focused on the discovery of bacterial diagnostic biomarkers for LC [61, 62].

These biomarker discovery studies often used saliva, sputum, bronchoscopic samples, or bronchoalveolar lavage fluid instead of direct lung biopsy, which is not performed on healthy subjects. However, lung tissue remains the most accurate sample to study lung microbiome alternations [63]. A study evaluating saliva microbiota revealed that bacterial profiles are significantly altered in LC patients compared to those from control subjects. In particular, Capnocytophaga, Selenomonas, and Veillonella were found to be more abundant in both lung squamous cell carcinoma and adenocarcinoma patients whereas Neisseria was less abundant than in the controls [64].

Another study compared bronchial brushing samples from cancerous site and contralateral noncancerous site of 24 LC patients and 18 healthy controls. The authors demonstrated that LC-associated microbiota profile is extremely divergent from that found in healthy subjects with a significant decrease in microbial diversity. More interestingly, the alterations of microbiota composition in unilateral lobe LC patients are extended to the contralateral noncancerous site suggesting a deep change of the whole lung microenvironment, which is linked to the development of LC [65].

Although increasing evidence has highlighted the key role of commensal microbiota in tumor-immune system interaction and treatment response, the main efforts have been focused on gut microbiota. Less is known on how lung microbiota could affect antitumor immunity and immunotherapy response.

Evidence suggests that manipulation of the composition of local flora may influence the ability of the host to generate an immune response that could mount both local and distal antitumor protective responses ameliorating the efficacy of immunotherapy treatment.

To date, several interesting clinical trials are attempted to study the role of lung microbiota on the efficacy of immunotherapy-based treatment in LC (Table 1).

An ongoing observational clinical trial (NCT03688347) at Iowa Institute of Human Genetics (Iowa, US) is currently recruiting patients with advanced or recurrent LC (and other solid tumors) that initiate a new line of immunotherapy, either alone or in combination with chemotherapy, targeted therapy, or other immunotherapy agents.

Recently, Stevenson et al. isolated and identified Enterococcus gallinarum MRx0518, a commensal Gram-positive species, demonstrating the antitumor efficacy of this bacteria strain in mouse models of different solid tumors, including LC. MRx0518, and more specifically its flagellin, acts on both the innate and the adaptive immune system showing strong immunostimulating properties. Its inactivation resulted in complete abrogation of the TLR5-mediated activation of NF- $\kappa B[66,67]$.

Based on these exciting results, the NCT03934827, a single center, open label clinical trial, is aimed at studying 


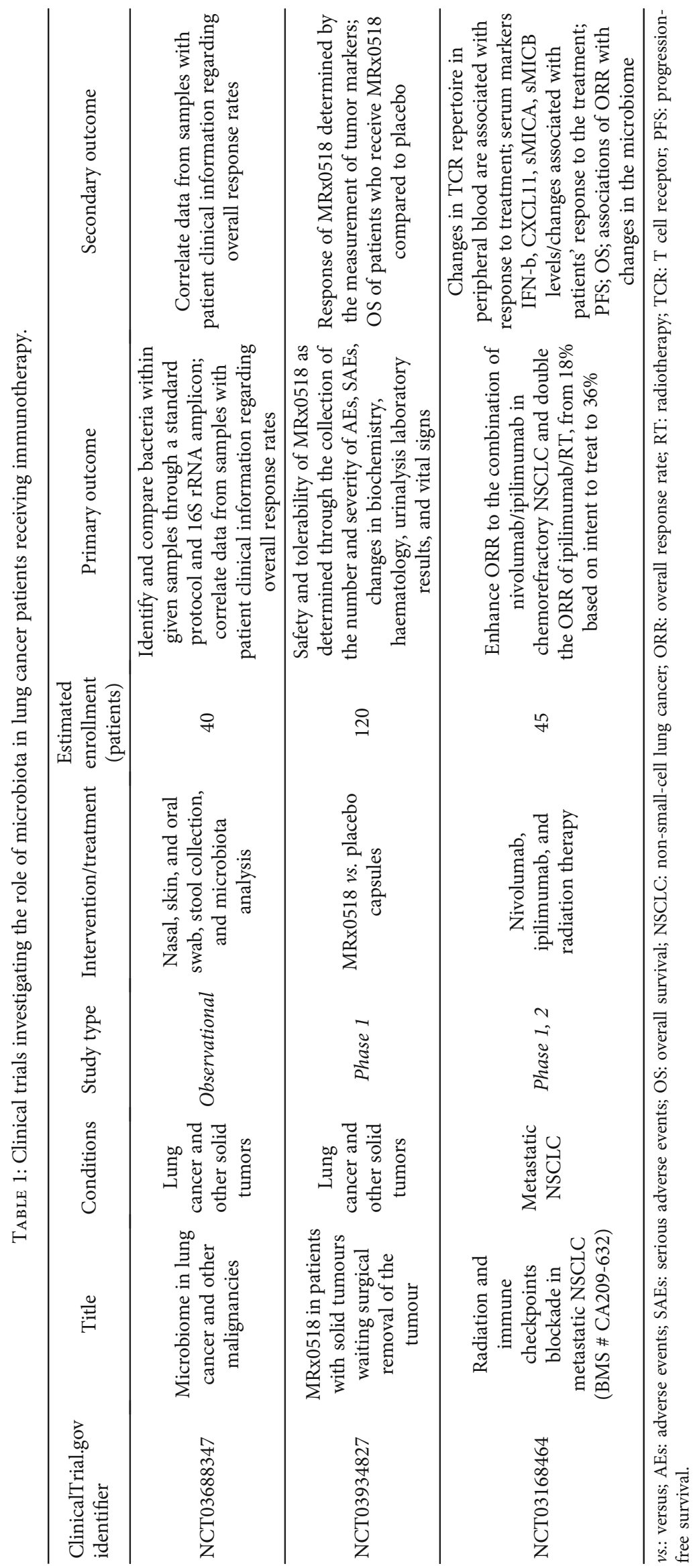




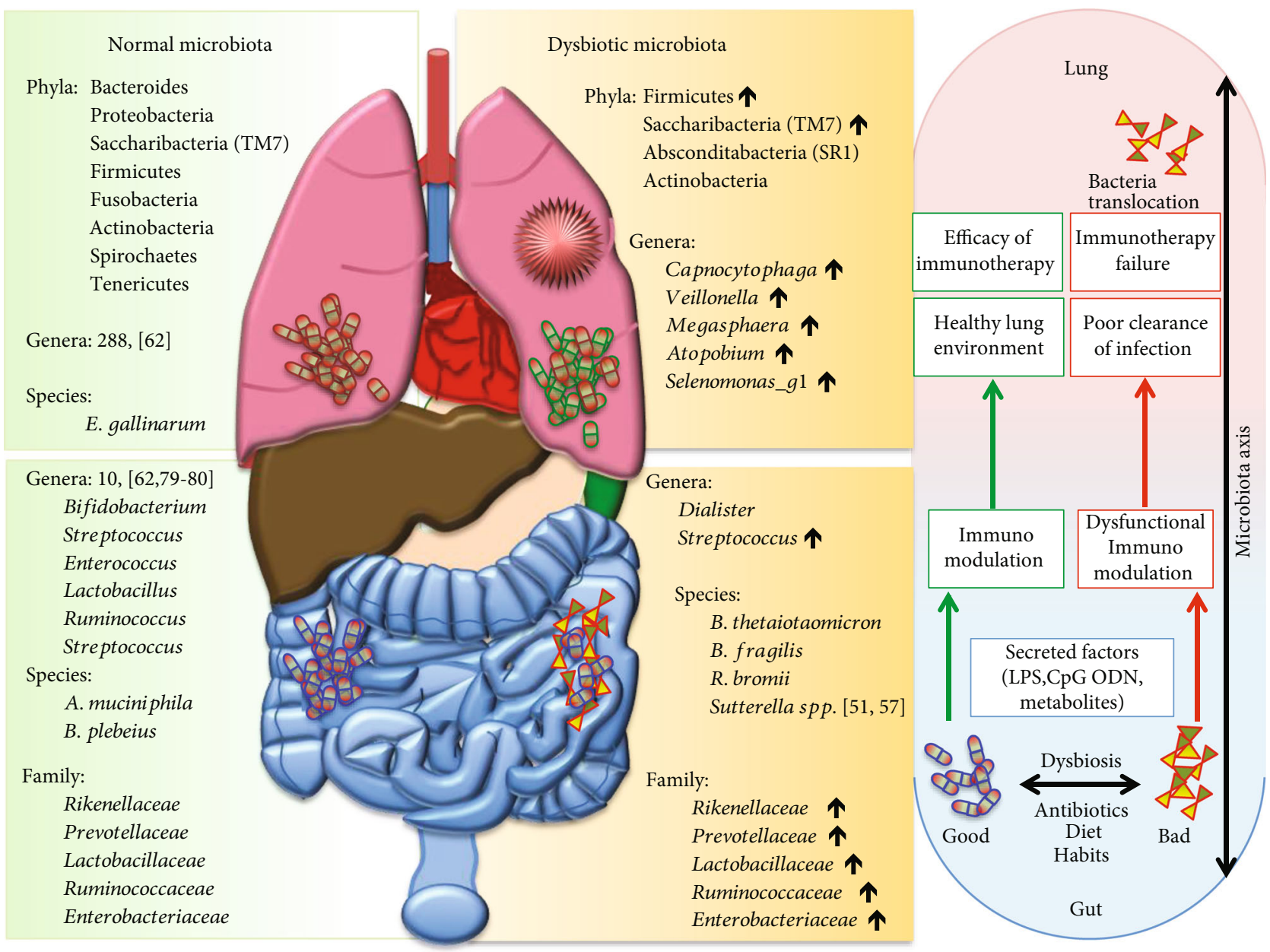

Microbiota

Lung cancer

A Enrichment

FIGURE 1: Increasing evidence supports the idea of a dynamic influence between host and microbiota. The fine line between human health and disease can be driven by friend (green) or foe (red) microbiota. We reported the main bacteria that could be responsible for the transition from a health to a pathological status. Commensal microorganisms are required for the maturation, education, and function of the immune system. A tight and continuous interaction of immune cells with microorganisms allows learning the difference between commensal and pathogenic bacteria that could influence immunotherapeutic treatment.

MRx0518 in combination with pembrolizumab in patients with LC and other solid tumors (at MD Anderson Cancer Center Houston, Texas, US). This study will assess the safety and tolerability and clinical benefit of MRx0518 in combination with pembrolizumab through the collection of adverse events.

Moreover, the NCT03168464 Interventional Clinical Trial at Weill Medical College of Cornell University (New York, US) is aimed at evaluating the association of ORR with changes in the microbiome in NSCLC patients with metastatic disease who have failed at least one prior treatment.

Although these studies are still in their infancy, they will provide a valid contribution in the exact determination of the role of the local microbiota in the response to immunotherapeutic agents and, on the other hand, will provide both new prognostic biomarkers and a powerful alternative tool to modulate the patient outcome.

\section{Gut-Lung Microbiota Axis}

The interaction between gut microbiota and host cells in the intestinal mucosa occurs in several ways. The pathogenassociated molecular patterns (PAMPs), provided by gut microbiota, serve as ligands for different Toll-like receptors (TLRs) on the surface of the intestinal epithelial cells (IECs). PAMPs from different microbiomal origin, such as lipopolysaccharide (LPS) or CpG ODN from bacteria, or viral double-stranded RNA, or toxin from parasites and fungi could activate TLR innate-adaptive immunity $[68,69]$ (Figure 1). In a similar way, also lipoteichoic acid (LTA), 
the main component of the Gram-positive cellular wall seems to function as potent immune activator with a signaling similar to the LPS activation pathway.

Indeed, the immune system through plasma cells and IgA secretion into the lumen of the gut could regulate in turn microbiota population [70]. Moreover, commensal bacteria and their metabolites (i.e., short-chain fatty acids (SCFAs) like butyrate, propionate, and acetate) directly stimulate IECs regulating immune cells. SCFAs might regulate the immune system through regulation of G-protein-coupled receptors (GPRs) and histone deacetylase [71], modulating epithelial and immune cell functions. Other cell types have also emerged as targets of SCFAs, including monocytes, dendritic cells, T cells, and intestinal epithelial cells [72].

In dendritic cells, treatment with SCFA butyrate is associated with decreased expression of the proinflammatory cytokines IL-12 and IFN- $\gamma$ and increased expression of Th2 cytokines [72]. Some evidence suggests that butyrate may regulate the ability of dendritic cells to present antigen and to prime $\mathrm{T}$ cells [73].

The gastrointestinal and respiratory tracts, although physically distant organs, are part of a shared mucosal immune ecosystem named the gut-lung axis [74]. Gut microbiota dysbiosis has been implicated in several lung diseases. Indeed, restoring microbiota in the gut of mice resulted in reduced severity of pneumonia [75].

It has been hypothesized a bidirectional crosstalk between the two microbiota entities which means that alteration of one compartment could impact on the other one.

This concept opens the possibility to indirectly modify lung bacterial composition, which represents the population physically close to lung tumor microenvironment, through gut microbiota modification strategies, such as fecal transplantation.

The dynamic crosstalk between the two compartments occurs through a direct translocation of bacteria from one to the other site or through the release into the bloodstream and the lymphatic system of bacteria-derived immunomodulatory molecules, which affect systemic immunity [75-80].

The massive crosstalk between the microbiota of gutlung axis and its decisive role in inflammation and against lung infections could open to new therapeutic and immunization strategies.

\section{Conclusions}

The straight interaction between microbiota and host epithelial barrier is required for the maturation, education, and function of the immune system impacting the host's health but also the power of immunotherapy to boost anticancer response. The molecular crosstalk between the gut and lung microbiota and anticancer immune regulation represents a novel area of research. Potentially, the microbiota could modulate and eventually potentiate an immune response by the release of proinflammatory cytokines, metabolites, or nucleic acids, allowing a microbiota-based selection of patients who could benefit from specific immunotherapy treatment.
However, microbiota composition differs widely according to host genetics and racial characteristic as well as diet and eating habits. These variables are closely related to geographical location, suggesting therefore the need of more in-depth clinical research studies, looking at ethnic diversity as well as eating habits and environment-related factors.

These substantial divergences in the basal microbiome components of different study populations question the universality of the microbiome-based findings and recommend taking into consideration more geographically tailored approaches [81]. Because this research area is still in its infancy, new efforts are necessary to determine the role of the microbiota in the response to immunotherapeutic agents and also to comprehensively illustrate the gut-lung axis and its implications.

\section{Conflicts of Interest}

E.B. received honoraria or speakers' fee from MSD, AstraZeneca, Celgene, Pfizer, Helsinn, Eli-Lilly, BMS, Novartis, and Roche; E.B. also received research grants from I.A.S.L.C. (International Association for the Study of Lung Cancer), L.I.L.T. (Lega Italiana per la Lotta contro i Tumori), Fondazione Cariverona, Astra-Zeneca, Roche, and Open Innovation, not related to the submitted work. S.P. reports personal fees from Astra-Zeneca, Eli-Lilly, BMS, Boehringer Ingelheim, Roche, MSD, and Istituto Gentili, outside the submitted work. G.T. reports grants and others from Celgene, Novartis, Roche, Incyte, and Merck Serono and grants from Fondazione Cariverona, outside the submitted work. The remaining authors have nothing to disclose.

\section{Authors' Contributions}

Giampaolo Tortora and Emilio Bria share the last coauthorship.

\section{Acknowledgments}

C.C., G.P., V. di N., E.D’A. E.V., M.G.F., S.P., G.T., and E.B. are currently supported by the Associazione Italiana Ricerca Cancro (AIRC 5x1000 21052, IG 18599, and IG 20583). E.B. is currently supported by the Institutional Funds of the Università Cattolica del Sacro Cuore (UCSC-Project D12018-2019).

\section{References}

[1] R. L. Siegel, K. D. Miller, and A. Jemal, "Cancer statistics, 2019," CA: a Cancer Journal for Clinicians, vol. 69, no. 1, pp. 7-34, 2019.

[2] Y. Miura and N. Sunaga, "Role of immunotherapy for oncogene-driven non-small cell lung cancer," Cancers, vol. 10, no. 8, p. 245, 2018.

[3] M. Reck, D. Rodríguez-Abreu, A. G. Robinson et al., "Pembrolizumab versus chemotherapy for PD-L1-positive non-smallcell lung cancer," The New England Journal of Medicine, vol. 375, no. 19, pp. 1823-1833, 2016.

[4] T. S. K. Mok, Y. L. Wu, I. Kudaba et al., "Pembrolizumab versus chemotherapy for previously untreated, PD-L1-expressing, 
locally advanced or metastatic non-small-cell lung cancer (KEYNOTE-042): a randomised, open-label, controlled, phase 3 trial," The Lancet, vol. 393, no. 10183, pp. 1819-1830, 2019.

[5] L. Paz-Ares, A. Luft, D. Vicente et al., "Pembrolizumab plus chemotherapy for squamous non-small-cell lung cancer," The New England Journal of Medicine, vol. 379, no. 21, pp. 2040-2051, 2018.

[6] C. J. Langer, S. M. Gadgeel, H. Borghaei et al., "Carboplatin and pemetrexed with or without pembrolizumab for advanced, non- squamous non-small-cell lung cancer: a randomised, phase 2 cohort of the open- label KEYNOTE-021 study," The Lancet Oncology, vol. 17, no. 11, pp. 1497-1508, 2016.

[7] L. Gandhi, D. Rodríguez-Abreu, S. Gadgeel et al., "Pembrolizumab plus chemotherapy in metastatic non-small-cell lung cancer," The New England Journal of Medicine, vol. 378, no. 22, pp. 2078-2092, 2018.

[8] Y. Oshima, T. Tanimoto, K. Yuji, and A. Tojo, "EGFR-TKIassociated interstitial pneumonitis in nivolumab-treated patients with non-small cell lung cancer," JAMA Oncology, vol. 4, no. 8, pp. 1112-1115, 2018.

[9] L. Horn, D. R. Spigel, E. E. Vokes et al., "Nivolumab versus docetaxel in previously treated patients with advanced nonsmall-cell lung cancer: two-year outcomes from two randomized, open-label, phase III trials (CheckMate 017 and CheckMate 057)," Journal of Clinical Oncology, vol. 35, no. 35, pp. 3924-3933, 2017.

[10] R. S. Herbst, P. Baas, D. W. Kim et al., "Pembrolizumab versus docetaxel for previously treated, PD-L1-positive, advanced non-small-cell lung cancer (KEYNOTE-010): a randomised controlled trial," The Lancet, vol. 387, no. 10027, pp. 15401550, 2016.

[11] A. Rittmeyer, F. Barlesi, D. Waterkamp et al., "Atezolizumab versus docetaxel in patients with previously treated non-smallcell lung cancer (OAK): a phase 3, open-label, multicentre randomised controlled trial," The Lancet, vol. 389, no. 10066, pp. 255-265, 2017.

[12] J. He, Y. Hu, M. Hu, and B. Li, "Development of PD-1/PD-L1 pathway in tumor immune microenvironment and treatment for non-small cell lung cancer," Scientific Reports, vol. 5, article 13110, 2015

[13] D. Kazandjian, S. Khozin, G. Blumenthal et al., "Benefit-risk summary of nivolumab for patients with metastatic squamous cell lung cancer after platinum-based chemotherapy: a report from the US Food and Drug Administration," JAMA Oncology, vol. 2, no. 1, pp. 118-122, 2016.

[14] L. Horn, M. Reck, and D. R. Spigel, "The future of immunotherapy in the treatment of small cell lung cancer," The Oncologist, vol. 21, no. 8, pp. 910-921, 2016.

[15] M. D. Hellmann, T. E. Ciuleanu, A. Pluzanski et al., "Nivolumab plus ipilimumab in lung cancer with a high tumor mutational burden," The New England Journal of Medicine, vol. 378, no. 22, pp. 2093-2104, 2018.

[16] S. Peters, B. C. Cho, N. Reinmuth et al., "Abstract CT074: tumor mutational burden (TMB) as a biomarker of survival in metastatic non-small cell lung cancer (mNSCLC): blood and tissue TMB analysis from MYSTIC, a phase III study of first-line durvalumab \pm tremelimumab vs chemotherapy," Cancer Research, vol. 79, no. 13, 2019.

[17] A. P. Bhatt, M. R. Redinbo, and S. J. Bultman, "The role of the microbiome in cancer development and therapy," CA: A Cancer Journal for Clinicians, vol. 67, no. 4, pp. 326-344, 2017.
[18] C. A. Thaiss, N. Zmora, M. Levy, and E. Elinav, "The microbiome and innate immunity," Nature, vol. 535, no. 7610, pp. 65-74, 2016.

[19] S. R. Gill, M. Pop, R. T. Deboy et al., "Metagenomic analysis of the human distal gut microbiome," Science, vol. 312, no. 5778, pp. 1355-1359, 2006.

[20] R. Bingula, M. Filaire, N. Radosevic-Robin et al., "Desired turbulence? Gut-lung axis, immunity, and lung cancer," Journal of Oncology, vol. 2017, Article ID 5035371, 15 pages, 2017.

[21] D. R. Samuelson, D. A. Welsh, and J. E. Shellito, "Regulation of lung immunity and host defense by the intestinal microbiota," Frontiers in Microbiology, vol. 6, article 1085, 2015.

[22] H. Renz, P. Brandtzaeg, and M. Hornef, "The impact of perinatal immune development on mucosal homeostasis and chronic inflammation," Nature Reviews Immunology, vol. 12, no. 1, pp. 9-23, 2011.

[23] T. Olszak, D. An, S. Zeissig et al., "Microbial exposure during early life has persistent effects on natural killer T cell function," Science, vol. 336, no. 6080, pp. 489-493, 2012.

[24] N. Iida, A. Dzutsev, C. A. Stewart et al., "Commensal bacteria control cancer response to therapy by modulating the tumor microenvironment," Science, vol. 342, no. 6161, pp. 967-970, 2013.

[25] A. Sivan, L. Corrales, N. Hubert et al., "Commensal Bifidobacterium promotes antitumor immunity and facilitates anti-PDL1 efficacy," Science, vol. 350, no. 6264, pp. 1084-1089, 2015.

[26] M. Vetizou, J. M. Pitt, R. Daillere et al., "Anticancer immunotherapy by CTLA- 4 blockade relies on the gut microbiota," Science, vol. 350, no. 6264, pp. 1079-1084, 2015.

[27] V. Matson, J. Fessler, R. Bao et al., "The commensal microbiome is associated with anti-PD-1 efficacy in metastatic melanoma patients," Science, vol. 359, no. 6371, pp. 104-108, 2018.

[28] E. van Nood, A. Vrieze, M. Nieuwdorp et al., "Duodenal infusion of donor feces for recurrent Clostridium difficile," The New England Journal of Medicine, vol. 368, no. 5, pp. 407415, 2013.

[29] P. Moayyedi, M. G. Surette, P. T. Kim et al., "Fecal microbiota transplantation induces remission in patients with active ulcerative colitis in a randomized controlled trial," Gastroenterology, vol. 149, no. 1, pp. 102-109.e6, 2015.

[30] N. G. Rossen, S. Fuentes, M. van der Spek et al., "Findings from a randomized controlled trial of fecal transplantation for patients with ulcerative colitis," Gastroenterology, vol. 149, no. 1, pp. 110-118.e4, 2015.

[31] S. Paramsothy, M. A. Kamm, N. O. Kaakoush et al., "Multidonor intensive faecal microbiota transplantation for active ulcerative colitis: a randomised placebo-controlled trial," The Lancet, vol. 389, no. 10075, pp. 1218-1228, 2017.

[32] A. C. Ford, "Stool as a treatment for IBS: more questions than answers?," The Lancet Gastroenterology \& Hepatology, vol. 3, no. 1, pp. 2-3, 2018.

[33] O. Goulet, "Potential role of the intestinal microbiota in programming health and disease," Nutrition Reviews, vol. 73, Supplement 1, pp. 32-40, 2015.

[34] Y. Belkaid and T. W. Hand, "Role of the microbiota in immunity and inflammation," Cell, vol. 157, no. 1, pp. 121-141, 2014.

[35] W. C. Kieper, A. Troy, J. T. Burghardt et al., "Recent immune status determines the source of antigens that drive homeostatic 
T cell expansion," The Journal of Immunology, vol. 174, no. 6 , pp. 3158-3163, 2005.

[36] M. Uribe-Herranz, K. Bittinger, S. Rafail et al., "Gut microbiota modulates adoptive cell therapy via CD $8 \alpha$ dendritic cells and IL-12," JCI Insight, vol. 3, no. 4, 2018.

[37] C. T. Fagundes, F. A. Amaral, A. T. Vieira et al., "Transient TLR activation restores inflammatory response and ability to control pulmonary bacterial infection in germfree mice," The Journal of Immunology, vol. 188, no. 3, pp. 1411-1420, 2012.

[38] R. Cianci, L. Franza, G. Schinzari et al., "The interplay between immunity and microbiota at intestinal immunological niche: the case of cancer," International Journal of Molecular Sciences, vol. 20, no. 3, p. 501, 2019.

[39] C. Abraham and J. H. Cho, "IL-23 and autoimmunity: new insights into the pathogenesis of inflammatory bowel disease," Annual Review of Medicine, vol. 60, pp. 97-110, 2009.

[40] F. J. Verdam, S. Fuentes, C. de Jonge et al., "Human intestinal microbiota composition is associated with local and systemic inflammation in obesity," Obesity, vol. 21, no. 12, pp. E607E615, 2013.

[41] Y. K. Lee, J. S. Menezes, Y. Umesaki, and S. K. Mazmanian, "Proinflammatory T-cell responses to gut microbiota promote experimental autoimmune encephalomyelitis," Proceedings of the National Academy of Sciences of the United States of America, vol. 108, Supplement 1, pp. 4615-4622, 2011.

[42] K. Atarashi, T. Tanoue, T. Shima et al., "Induction of colonic regulatory T cells by indigenous Clostridium species," Science, vol. 331, no. 6015, pp. 337-341, 2011.

[43] L. Carbognin, S. Pilotto, R. Nortilli et al., "Predictive and prognostic role of tumor-infiltrating lymphocytes for early breast cancer according to disease subtypes: sensitivity analysis of randomized trials in adjuvant and neoadjuvant setting," The Oncologist, vol. 21, no. 3, pp. 283-291, 2016.

[44] W. Li, Y. Deng, Q. Chu, and P. Zhang, "Gut microbiome and cancer immunotherapy," Cancer Letters, vol. 447, pp. 41-47, 2019.

[45] S. Raju, R. Joseph, and S. Sehgal, "Review of checkpoint immunotherapy for the management of non-small cell lung cancer," ImmunoTargets and Therapy, vol. 7, pp. 63-75, 2018.

[46] R. Ferrara, L. Mezquita, M. Texier et al., "Hyperprogressive disease in patients with advanced non-small cell lung cancer treated with PD-1/PD-L1 inhibitors or with single-agent chemotherapy," JAMA Oncology, vol. 4, no. 11, pp. 1543-1552, 2018.

[47] L. Carbognin, S. Pilotto, M. Milella et al., "Differential activity of nivolumab, pembrolizumab and MPDL3280A according to the tumor expression of programmed death-ligand-1 (PD-L1): sensitivity analysis of trials in melanoma, lung and genitourinary cancers," PLoS One, vol. 10, no. 6, article e0130142, 2015.

[48] B. Routy, E. le Chatelier, L. Derosa et al., "Gut microbiome influences efficacy of PD-1-based immunotherapy against epithelial tumors," Science, vol. 359, no. 6371, pp. 91-97, 2018.

[49] Y. Jin, H. Dong, L. Xia et al., "The diversity of gut microbiome is associated with favorable responses to anti-PD-1 immunotherapy in Chinese non-small cell lung cancer patients," Journal of Thoracic Oncology, vol. 14, no. 10, 2019.

[50] T. Hakozaki, Y. Okuma, M. Omori, and Y. Hosomi, "Impact of prior antibiotic use on the efficacy of nivolumab for non-small cell lung cancer," Oncology Letters, vol. 17, no. 3, pp. 29462952, 2019.
[51] L. P. Andrea Botticelli, I. Zizzari, F. Del Chierico et al., "Changes of microbiome profile during nivolumab treatment in NSCLC patients," Journal of Clinical Oncology, vol. 36, p. 15, 2018.

[52] V. Cortez-Retamozo, M. Etzrodt, A. Newton et al., "Origins of tumor-associated macrophages and neutrophils," Proceedings of the National Academy of Sciences of the United States of America, vol. 109, no. 7, pp. 2491-2496, 2012.

[53] S. Viaud, F. Saccheri, G. Mignot et al., "The intestinal microbiota modulates the anticancer immune effects of cyclophosphamide," Science, vol. 342, no. 6161, pp. 971-976, 2013.

[54] E. S. Gollwitzer, S. Saglani, A. Trompette et al., "Lung microbiota promotes tolerance to allergens in neonates via PD-L1," Nature Medicine, vol. 20, no. 6, pp. 642-647, 2014.

[55] D. N. O'Dwyer, R. P. Dickson, and B. B. Moore, "The lung microbiome, immunity, and the pathogenesis of chronic lung disease," The Journal of Immunology, vol. 196, no. 12, pp. 4839-4847, 2016.

[56] J. R. Erb-Downward, D. L. Thompson, M. K. Han et al., “Analysis of the lung microbiome in the "healthy" smoker and in COPD," PLoS One, vol. 6, no. 2, article e16384, 2011.

[57] K. L. Greathouse, J. R. White, A. J. Vargas et al., "Interaction between the microbiome and TP53 in human lung cancer," Genome Biology, vol. 19, no. 1, p. 123, 2018.

[58] B. Boursi, R. Mamtani, K. Haynes, and Y. X. Yang, "Recurrent antibiotic exposure may promote cancer formation - another step in understanding the role of the human microbiota?," European Journal of Cancer, vol. 51, no. 17, pp. 2655-2664, 2015.

[59] R. P. Dickson, F. J. Martinez, and G. B. Huffnagle, "The role of the microbiome in exacerbations of chronic lung diseases," The Lancet, vol. 384, no. 9944, pp. 691-702, 2014.

[60] C. Jin, G. K. Lagoudas, C. Zhao et al., "Commensal microbiota promote lung cancer development via $\gamma \delta \mathrm{T}$ cells," Cell, vol. 176, no. 5, pp. 998-1013.e16, 2019.

[61] S. J. S. Cameron, K. E. Lewis, S. A. Huws et al., "A pilot study using metagenomic sequencing of the sputum microbiome suggests potential bacterial biomarkers for lung cancer," PLoS One, vol. 12, no. 5, article e0177062, 2017.

[62] S. H. Lee, J. Y. Sung, D. Yong et al., "Characterization of microbiome in bronchoalveolar lavage fluid of patients with lung cancer comparing with benign mass like lesions," Lung Cancer, vol. 102, pp. 89-95, 2016.

[63] Q. Mao, F. Jiang, R. Yin et al., "Interplay between the lung microbiome and lung cancer," Cancer Letters, vol. 415, pp. 40-48, 2018.

[64] X. Yan, M. Yang, J. Liu et al., "Discovery and validation of potential bacterial biomarkers for lung cancer," American Journal of Cancer Research, vol. 5, no. 10, pp. 3111-3122, 2015.

[65] H. X. Liu, L. L. Tao, J. Zhang et al., "Difference of lower airway microbiome in bilateral protected specimen brush between lung cancer patients with unilateral lobar masses and control subjects," International Journal of Cancer, vol. 142, no. 4, pp. 769-778, 2018.

[66] D. L. Lauté-Caly, E. J. Raftis, P. Cowie et al., "The flagellin of candidate live biotherapeutic Enterococcus gallinarum MRx0518 is a potent immunostimulant," Scientific Reports, vol. 9, no. 1, p. 801, 2019.

[67] A. P. Alex Stevenson, A. Holt, D. L. Caly et al., "Host-microbe interactions mediating antitumorigenic effects of MRX0518, a gut microbiota-derived bacterial strain, in breast, renal and 
lung carcinoma," Journal of Clinical Oncology, vol. 36, article e15006, 2018.

[68] S. Akira, S. Uematsu, and O. Takeuchi, "Pathogen recognition and innate immunity," Cell, vol. 124, no. 4, pp. 783-801, 2006.

[69] P. G. Fallon and A. Alcami, "Pathogen-derived immunomodulatory molecules: future immunotherapeutics?" Trends in Immunology, vol. 27, no. 10, pp. 470-476, 2006.

[70] A. Cerutti and M. Rescigno, "The biology of intestinal immunoglobulin A responses," Immunity, vol. 28, no. 6, pp. 740750, 2008.

[71] J. R. Brestoff and D. Artis, "Commensal bacteria at the interface of host metabolism and the immune system," Nature Immunology, vol. 14, no. 7, pp. 676-684, 2013.

[72] A. J. Bilotta and Y. Cong, "Gut microbiota metabolite regulation of host defenses at mucosal surfaces: implication in precision medicine," Precision Clinical Medicine, vol. 2, no. 2, pp. 110-119, 2019.

[73] M. Sun, W. Wu, L. Chen et al., "Microbiota-derived shortchain fatty acids promote Th1 cell IL-10 production to maintain intestinal homeostasis," Nature Communications, vol. 9, no. 1, article 3555, 2018.

[74] K. F. Budden, S. L. Gellatly, D. L. Wood et al., "Emerging pathogenic links between microbiota and the gut-lung axis," Nature Reviews Microbiology, vol. 15, no. 1, pp. 55-63, 2017.

[75] T. J. Schuijt, J. M. Lankelma, B. P. Scicluna et al., "The gut microbiota plays a protective role in the host defence against pneumococcal pneumonia," Gut, vol. 65, no. 4, pp. 575-583, 2016.

[76] K. K. Barfod, M. Roggenbuck, L. Hansen et al., "The murine lung microbiome in relation to the intestinal and vaginal bacterial communities," BMC Microbiology, vol. 13, p. 303, 2013.

[77] A. J. Adami and J. L. Cervantes, "The microbiome at the pulmonary alveolar niche and its role in Mycobacterium tuberculosis infection," Tuberculosis, vol. 95, no. 6, pp. 651-658, 2015.

[78] J. Cervantes and B.-y. Hong, "The gut-lung axis in tuberculosis," Pathogens and Disease, vol. 75, no. 8, 2018.

[79] Y. Vallès, A. Artacho, A. Pascual-García et al., "Microbial succession in the gut: directional trends of taxonomic and functional change in a birth cohort of Spanish infants," PLoS Genetics, vol. 10, no. 6, article e1004406, 2014.

[80] C. Milani, S. Duranti, F. Bottacini et al., "The first microbial colonizers of the human gut: composition, activities, and health implications of the infant gut microbiota," Microbiology and Molecular Biology Reviews, vol. 81, no. 4, 2017.

[81] V. K. Gupta, S. Paul, and C. Dutta, "Geography, ethnicity or subsistence-specific variations in human microbiome composition and diversity," Frontiers in Microbiology, vol. 8, article 1162, 2017. 


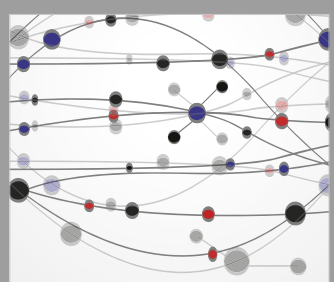

The Scientific World Journal
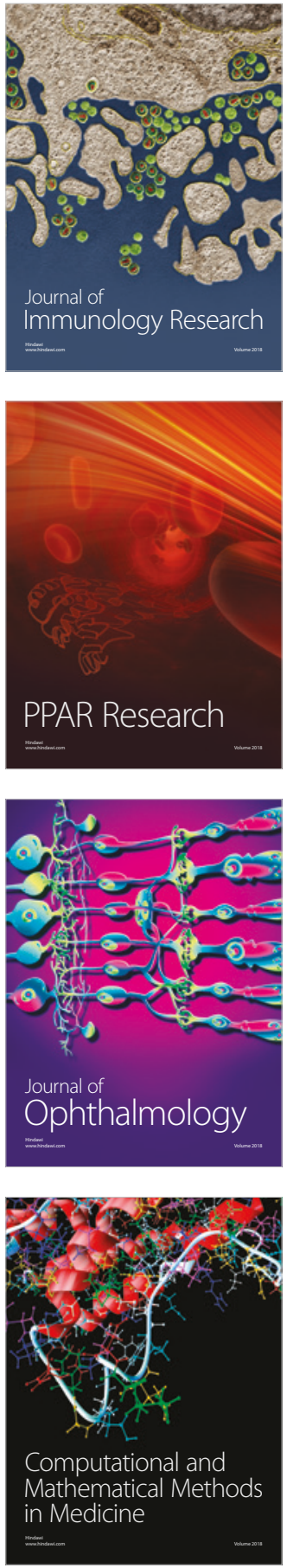

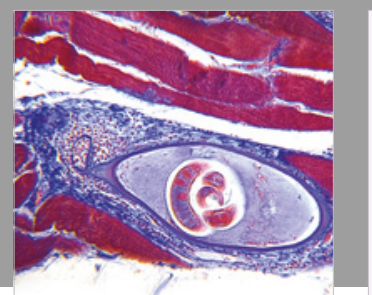

Gastroenterology Research and Practice

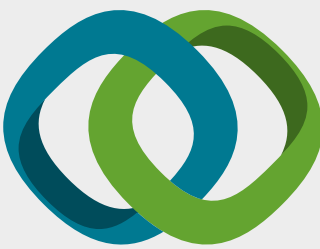

\section{Hindawi}

Submit your manuscripts at

www.hindawi.com
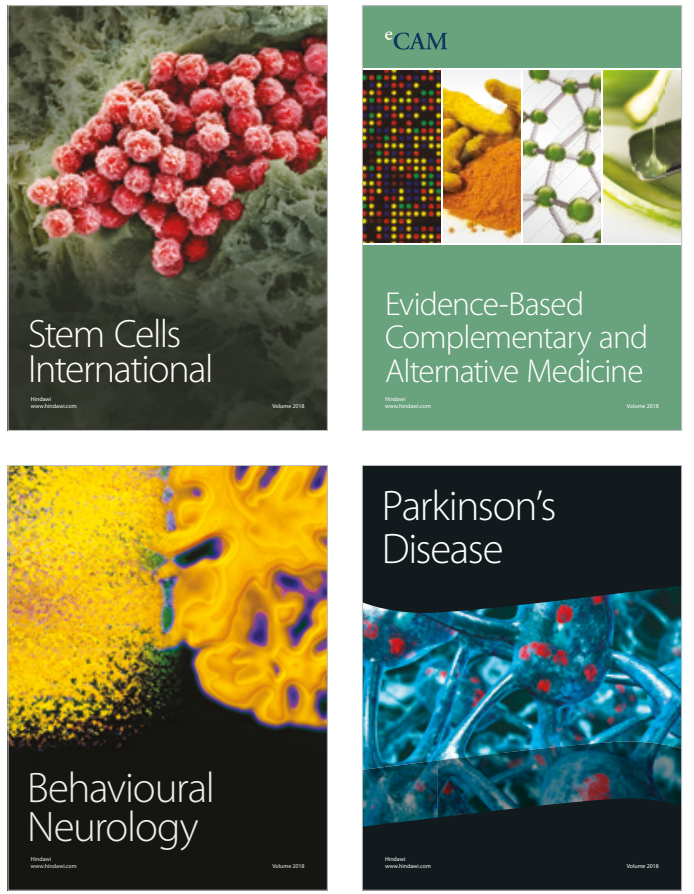

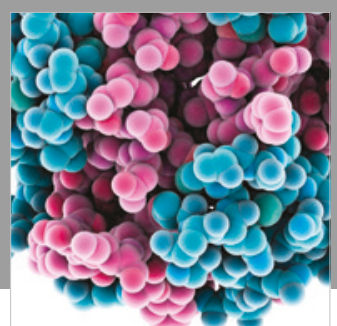

ournal of

Diabetes Research

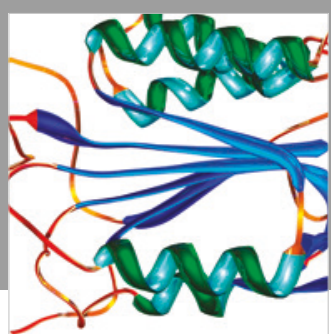

Disease Markers
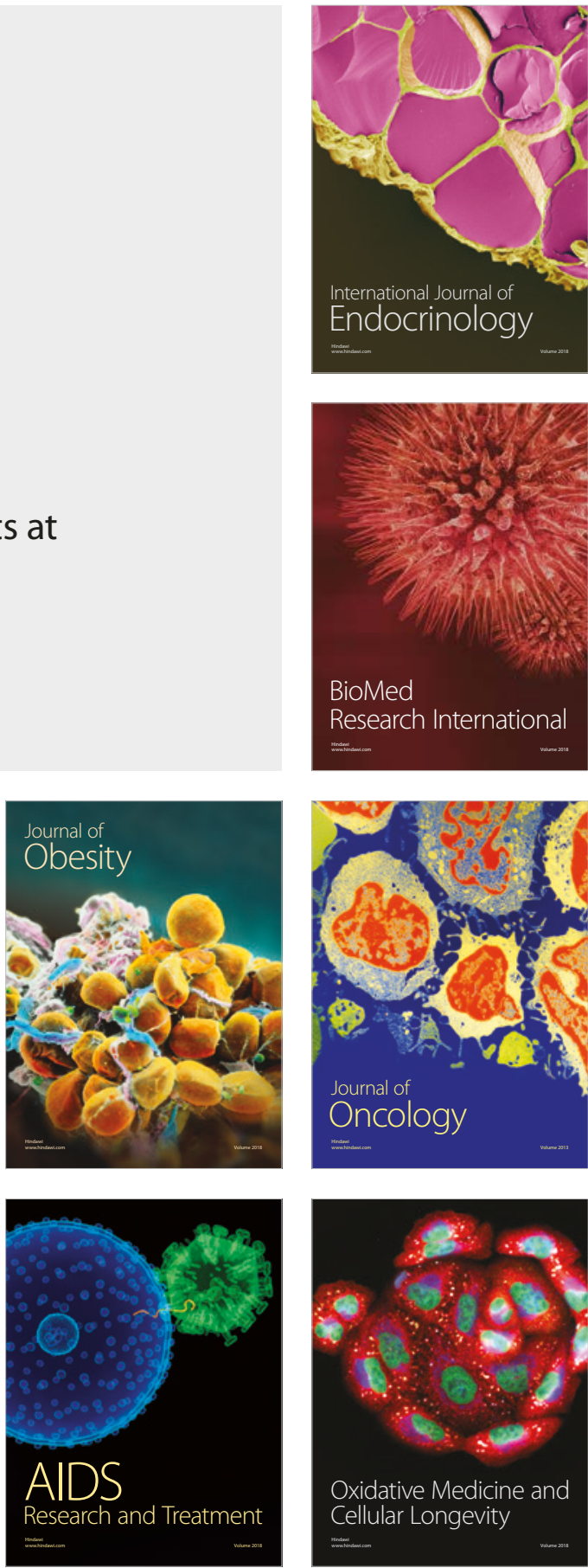